\title{
Dynamic Evolution Game of Travelers' Air-to-HSR Choice under the Scenario of HSR Speed-Up
}

\author{
Zhenhua Mou (D), ${ }^{1,2}$ Kangli Yan, ${ }^{1}$ Kepeng Li, ${ }^{1}$ Dongfu Shen, ${ }^{1}$ Xiang Li, ${ }^{1}$ Hanbing Wang, \\ Jijie Guo, ${ }^{3}$ and Yanyan Chen $\mathbb{D}^{2}$
}

\author{
${ }^{1}$ School of Transportation Engineering, Shandong Jianzhu University, Jinan 250101, China \\ ${ }^{2}$ Beijing Key Laboratory of Traffic Engineering, Beijing University of Technology, Beijing 100124, China \\ ${ }^{3}$ Jinan Rail Transit Group Co., Ltd., Jinan 250101, China
}

Correspondence should be addressed to Yanyan Chen; cdyan@bjut.edu.cn

Received 25 May 2020; Revised 23 December 2020; Accepted 2 January 2021; Published 16 January 2021

Academic Editor: Libor Pekař

Copyright ( 92021 Zhenhua Mou et al. This is an open access article distributed under the Creative Commons Attribution License, which permits unrestricted use, distribution, and reproduction in any medium, provided the original work is properly cited.

With the technological advancement, China's high-speed rail (HSR) has achieved a higher running speed than ever before, which at the same time leads to the intensive competition between air and high-speed rail with trips within $500-1000 \mathrm{~km}$, as well as to the significant changes of travelers' air-to-HSR choice. In order to explore the dynamic adaptation of travelers' choice of high-speed rail (HSR) and air in the case of HSR speed-up, a logit dynamics model of travelers' choice based on evolutionary game was constructed to describe the evolution of travelers' choice. With HSR-air travel time and the ticket price of origin-destination (OD) points of Beijing-Shanghai as the benchmark, the study set up two scenarios of HSR speed-up and compared and analyzed their payoffs in value. On the basis of mathematical simulation, the travelers' "air-to-HSR choice" and the change of competition range under the background of HSR speed-up were both discussed. The result indicated that the HSR speed-up would attract more travelers, and its share rate would rise to $58.31 \%$ and $72.73 \%$, respectively, with HSR running speed increasing to 400 and $600 \mathrm{~km} /$ $\mathrm{h}$; the marginal utility of HSR speed-up is more obvious, and in the meantime, the increase of HSR running speed extends the intense HSR-air competition range to longer-distance travels. In the case of HSR speed-up, travelers' choice changes as well. The HSR operators themselves should formulate reasonable ticket prices and improve their management. At the same time, the air operators should adjust their operation plan in time.

\section{Introduction}

At present, considering the resource allocation of longdistance travel in China, high-speed rail (HSR) and air travel have held a dominant position. Though HSR development started relatively late compared with air travel, its improvement remains rapid. Since the Beijing-Shanghai HighSpeed Railway was opened more than 10 years ago, China's high-speed railway network has expanded to 35000 kilometers, and the passenger flow has reached 2.29 billion, which indicates that HSR has become the main competitor of air [1]. Empirical analysis shows that during the rapid development of high-speed rail, airlines' ticket prices, routes, and profits have been negatively affected [2-4]. For example, in France, air traffic has dropped by $50 \%$ between Paris and
Lyon after several years of HSR operation [5]; in Korea, air transportation demand has reduced by $34 \%$ to $75 \%$ between Seoul and Daegu by the stated preference model calibration [6]. In this case, we analyzed the dynamic adaptation process of travelers' air-to-HSR choice under different HSR speedup scenarios and then studied the new change of competition range between HSR and air.

From the perspective of the development of the two systems, the transportation speed of the air system has been kept at $800 \mathrm{~km} / \mathrm{h}$ and above, while the speed of the railway system has accelerated from $120 \mathrm{~km} / \mathrm{h}$ in the general-speed-railway era to $350 \mathrm{~km} / \mathrm{h}$ in the high-speed-railway era due to continuously upgrading technologies, and the latest trial shows that the highest trial speed of China's HSR can reach $605 \mathrm{~km} / \mathrm{h}$. According to the analysis of historical data, the fierce 
competition between HSR and air may occur within $1000 \mathrm{~km}$, especially within $400-800 \mathrm{~km} / \mathrm{h}$, so when the HSR speed reaches $605 \mathrm{~km} / \mathrm{h}[7,8]$, what changes will happen to the new round of competition range and what kind of adaptation will travelers face when they make choices? To answer these questions, with the travel time and ticket price of origindestination (OD) points of Beijing-Shanghai HSR and air as the reference benchmark, this paper proposed a logit dynamic evolution model to analyze the dynamic adaptation process of travelers' air-to-HSR choice under different HSR speed-up scenarios and further analyzed the competition range of HSR and air through the logit allocation model.

At present, most research studies about China's HSR network focus on the influence of the HSR network on local and regional economy $[9,10]$, urban development [11], and the aggregation mode of service industry in urban areas [12]. The research studies about HSR and air mainly focus on the impact of HSR development on air and the cooperationcompetition development of HSR and air through data investigation, simulation evaluation, and specific case studies [6, 13-15]. Many research results have been obtained through the study of the travelers' choice of travel mode in the HSR and air competition environment, and the ticket price and travel time were considered as the main factors influencing the travelers' travel mode choices [16, 17], followed by flight frequency and road-feeder service time [2]. Wen et al. further pointed out that some HSR passengers are more sensitive to the pretrip road-feeder charge than the time [18]. The logit discrete model is adopted as the main research method, and it is assumed that the decision-maker is rational and has relevant experience, which is a classical discrete model based on random utility theory [19]. With the deepening of research, an application system has been formed with binary logit, multinomial logit, nested logit, and mixed logit as the main part [20]. This theory has further proved that the ticket price is the main factor affecting travelers' air-to-HSR choice [21], and travel purpose is another important factor affecting the travel mode choice of the travelers [22].

Different from existing studies, this paper addresses the issue of the dynamic development direction and process of travelers' choice changes. In fact, travel mode choosing behavior is not a static process. Travelers often demonstrate limited rationality with incomplete information, value differences, and other factors. They will estimate the traffic volume and cost of different travel modes according to empirical utility [23]. When the traffic environment changes, travelers' choosing behavior of traffic mode needs a period of dynamic adaptation to achieve the optimal stable equilibrium state. Evolutionary game, as a dynamic model, can well describe the dynamic selection process of travelers and explain the game phenomenon, and it can analyze the evolution process and evolution result of travelers' travel mode selection under the scenarios of HSR speed-up $[24,25]$.

Based on the development of classical game theory, Hotelling model, Cournot model, Bertrand model, and duopoly model have formed a solid theoretical foundation in solving the game problems such as analyzing the change of competition range $[7,26,27]$. Based on evolutionary game theory, the transfer effect of ticket price on travelers' travel mode and the relationship between HSR, air, and travelers' welfare have been discussed by many scholars [28, 29]. The case in Europe shows that the existing revenue mechanism of the market of the medium- and long-distance transportation has changed due to the HSR-air competition [15]; the Chinese case shows that the increase of HSR speed has a significant impact on air transport [30].

In the dynamic models that simulate the learning and strategies of game players, replication selection mechanism is the most common one [31]. Based on this, the study has found out that the HSR-air competition has a significant impact on the ticket price and market share of both sides [32], which is further shown in the three major aviation data reports in China [33]; similarly, the data also show that the ticket price and the frequency are the two main factors that lead to the decrease of civil aviation market share [17]; in the process of strategy selection, when the individual travel utility has certain constraints, the system model has a unique solution.

The empirical utility of participants has been considered in the study of travelers' travel mode selection under air and HSR competition, but the model analysis has focused more on the static situation and ignored the traveler's dynamic strategy. However, the replication selection mechanism, as a dynamic theoretical model of evolutionary game, has ignored the empirical utility of travelers. Besides, the analysis of HSR-air competition range needs to be specified. Based on the above analysis, the main contributions of this study can be summarized as follows:

(1) An innovative logit dynamic evolutionary game model has started the first try for travelers' travel mode selection under high-speed rail (HSR) speedup, considering the individual random utility maximization and empirical utility.

(2) A study has been launched on the change of HSR-air competition range in the transportation distance. The proposed sharing ratio of travelers of air and HSR at different operating speeds, which is based on HSR-air travel time and the ticket price of origindestination (OD) points of Beijing-Shanghai, and the change of HSR-air competition range in the transportation distance are further analyzed.

The rest part of the paper is arranged as follows: Section 2 introduces the establishment of logit dynamic model; Section 3 discusses the logit dynamic model evolution and stability analysis; Section 4 discusses the dynamic evolution of travelers' travel mode and analyzes the HSR-air competition range; and finally, Section 5 summarizes the research findings and limitations.

\section{Methodology}

2.1. Establishment of Game Model. The study assumes that travelers can only choose the travel mode of HSR and air between the same origin-destination (OD) points, and the total demand of long-distance travelers between the same 
OD is $N(N>0)$. Since the number of travelers in the traffic network is infinite, the travelers' travel mode selection can be regarded as a group evolutionary game problem [34]. In order to model and analyze it, an assumption of the game elements can be proposed as follows:

(1) Taking the total number of travelers between the same OD points as a single group, assuming that total travel demand of the group is $N$ (in order to simplify the calculation, the sum of HSR and air demand can be assumed as a unit 1).

(2) The selection strategy set is all the strategies that the players can choose in the game. According to Xiao's research, it is assumed that the pure strategy of the OD points $M=\{\operatorname{Air}(A), \operatorname{HSR}(R)\}$ [34].

(3) In this game, only HSR and air are available. Assuming that the proportion of travelers choosing air travel is $x$, and the probability of choosing HSR is $1-x[35]$.

(4) $\pi_{i j}$ indicates the payoff when the opponent adopts $j$ travel mode and the game player chooses $i$ travel mode $(i=1,2 ; j=1,2)$ ("opponent" is another term naming the game player in the evolutionary game. In this paper, opponent can be understood as follows: if the traveler chooses between HSR and air, some travelers are named as game player $\mathrm{A}$, and the rest is named as game player $\mathrm{B}$, and then $\mathrm{B}$ can be understood as A's opponent) [35].

Since travelers belong to the same type of the income group and the time cost is same, the game is symmetric. Travelers can only choose one strategy at a time. In each travel case, both sides of the game do not know the opponent's decision-making and its payoff and can only surmise the next travel plan through continuous trial and error. Therefore, the payoff matrix of evolutionary game for travel mode selection is shown in Table 1.

When both traveler and opponent adopt air travel, it is usually at the peak season for travel, and air ticket prices are at a high level, but the obtained payoff by both parties in the game that achieved their travel purposes is relatively high, and the payoff can be indicated as $\pi_{11}$; when opponents choose HSR and travelers choose air travel, the original airpassenger flow decreases, and the operator regulates the ticket price. At this time, the payoff of travelers choosing air travel is $\pi_{12}$. At present, the ticket price of HSR does not change much throughout the year between the same OD points, so no matter what kind of strategies the opponent adopts, the payoff of travelers choosing HSR is always $\pi_{2}$.

With mixed strategy $(x, 1-x), 0 \leq x \leq 1$, the expected payoff functions [36] of travelers choosing air and HSR travel are as follows:

$$
\begin{aligned}
E_{1}(x) & =\pi_{11} x+\pi_{12}(1-x), \\
E_{2}(x) & =\pi_{2} x+\pi_{2}(1-x), \\
\bar{E} & =x E_{1}(x)+(1-x) E_{2}(x),
\end{aligned}
$$

TABLE 1: Payoff matrix of the game of travel mode choices.

\begin{tabular}{lcc}
\hline Travel mode & Air & High-speed rail \\
\hline Air & $\left(\pi_{11}, \pi_{11}\right)$ & $\left(\pi_{12}, \pi_{2}\right)$ \\
High-speed rail & $\left(\pi_{2}, \pi_{12}\right)$ & $\left(\pi_{2}, \pi_{2}\right)$ \\
\hline
\end{tabular}

where $E_{1}(x)$ and $E_{2}(x)$ indicate the expected payoff functions of choosing air and HSR travel, respectively.

2.2. Mixed Strategy Nash Equilibrium. Mixed strategy Nash equilibrium is a rational strategy directing against the uncertainty of other players' choice. Its main feature is that each pure strategy as a part of mixed strategy has the same expectation value [37]. Otherwise, a player will choose the strategy with the highest expectation and exclude all other strategies, which means that the original state is not an equilibrium. According to the definition of the state of mixed Nash equilibrium, under the two optional modes of HSR and air, if the mixed strategy $\left(x^{*}, 1-x^{*}\right)$ makes the following inequality hold, then the hybrid strategy will be seemed as the Nash equilibrium [38]:

$$
\begin{array}{ll}
E_{1}\left(x^{*}\right) \geq E_{1}(x), & \forall_{x} \in[0,1], \\
E_{2}\left(x^{*}\right) \geq E_{2}(x), & \forall_{x} \in[0,1] .
\end{array}
$$

If the two revenue functions of the mixed strategy are equal, we can get the following result:

$$
E_{1}\left(x^{*}\right)=E_{2}\left(x^{*}\right), \quad x^{*} \in[0,1] .
$$

Furthermore, we can obtain the Nash equilibrium expression of the hybrid strategy:

$$
x^{*}=\frac{\pi_{2}-\pi_{12}}{\pi_{11}-\pi_{12}} .
$$

\subsection{Average Dynamic Evolution Process of Travel Mode} Selection. Based on the evolutionary game, travelers will adjust their strategies in the process of travel mode selection. If the adjustment trajectory can converge to the Nash equilibrium point, it shows that the equilibrium point is stable; otherwise, it is not stable. There is no guarantee that the system will be stable in the optimal equilibrium state. The evolution of travelers' selection of travel modes will be deduced in the following parts.

In practice, the actor is not absolutely rational but is limited by bounded rationality. The HSR and air game leads to changes in travel costs of travelers under HSR speed-up, and the travel decision-making changes and travel mode adaptation process meet the inertia and nearsightedness principles [34]. Inertia can be understood as travelers only occasionally reconsider their choices; nearsightedness means travelers modify their choices based on their current behavior and demands.

First of all, we take into account the probability expectation value of travelers changing travel mode in infinitesimal unit time $\mathrm{d} t$ under single OD pair traffic network 
environment with constant total travel demand. Assuming that the total travel demand between OD pair is constant $q$, the set of alternative modes between the two places is $M$. According to the principle of inertia, the time for each traveler to obtain the opportunity of decision revision obeys the $\lambda$ exponential distribution of the parameter [34]. The time distribution of decision revision opportunity for different travelers is independent of each other. Therefore, each traveler can expect $\lambda \mathrm{d} t$ chances to consider whether to revise the decision in the next unit interval $\mathrm{d} t$. That is to say, at any time, the probability of a traveler getting a decision-making correction opportunity is as follows:

$$
\frac{1}{\lambda}
$$

The probability of keeping the original decision (i.e., no chance of decision revision) is given as follows:

$$
\frac{\lambda-1}{\lambda}
$$

It is further pointed out that the current mode of transportation chosen by travelers is recorded as $i \in M$. For the travelers who have the opportunity of decision revision, the probability of switching from the current travel mode $i$ to the other mode $j$ is seen as the conditional conversion probability, which can be expressed by $\rho_{i j}$. According to the principle of nearsightedness, conditional conversion probability $\rho_{i j}$ is a function of the utility $E_{i}$ of the current travel mode and the sharing rate $X$ of the network mode. Assuming that the time distribution of decision revision and decision revision opportunity is independent, then for any traveler whose current travel mode is $i$, the probability of obtaining decision modification opportunity and changing travel mode to $j$ at any time is $\left(\rho_{i j} / \lambda\right)$, and the probability of maintaining the original travel mode $i$ is $\left(\rho_{i i} / \lambda\right)$. The relationship is as follows

$$
\frac{\rho_{i j}}{\lambda}+\frac{\rho_{i i}}{\lambda}=1
$$

The number of decision revision opportunities obtained by individuals within the time interval $[0, t]$ obeys the expected $\lambda t$ Poisson distribution. If the current mode share ratio is $X=\{x, i \in M\}$ and $x_{i}$ indicates the probability of travelers choosing the travel mode $i$, then the number of all travelers with the current travel mode $i$ is $q x_{i}$, and the number of decision revision chances obtained by these travelers in the unit time interval is

$$
q x_{i} \lambda \mathrm{d} t
$$

Multiplying the number of decision revision chances by the probability of decision revision, the expected value of travelers who change from travel mode $i$ to travel mode $j$ in the next unit interval $\mathrm{d} t$ is

$$
q x_{i} \rho_{i j} \mathrm{~d} t=q x_{i} \lambda \mathrm{d} t \frac{\rho_{i j}}{\lambda} .
$$

Furthermore, it can be obtained that in the next unit interval $\mathrm{d} t$, the expected value of the change in the number of travelers who choose the travel mode $i$ is

$$
q \times \mathrm{d} x_{i}=q \times\left(\sum_{j \in M} x_{j} \rho_{j i} \mathrm{~d} t-\sum_{j \in M} x_{i} \rho_{i j} \mathrm{~d} t\right)
$$

where $\rho_{i j}$ represents the probability of changing from the travel mode $j$ to the travel mode $i$.

Then, we can get the continuous differential equation of average dynamic change of travel mode share rate as follows [38]:

$$
f_{x_{i}}^{\prime}=\frac{\mathrm{d} x_{i}}{\mathrm{~d} t}=\sum_{j \in M} x_{j} \rho_{j i}-\sum_{j \in M} x_{i} \rho_{i j},
$$

where $f_{x_{i}}^{\prime}$ represents the change rate of the probability of the traveler's reselection $i$ in the next time state when the current travel mode $i$ is selected.

2.4. Establishment of the Logit Dynamic Game Model for Travel Mode Selection. It is assumed that travelers do not consider the choice of other travelers but make decisions based on the maximum principle of empirical utility and individual random utility. In this case, the conditional transformation probability $\rho_{i j}$ conforms to logit discrete mechanism. Due to the difference and independence between HSR and air, the conditional conversion probability $\rho_{i j}$ of travelers from current travel mode $i$ to travel mode $j$ is equal to the probability of travel mode $j$ being selected in the multiple logit model [19]:

$$
\rho_{i j}=\frac{e^{E_{j}}}{\sum_{x \in M}\left(E_{x}\right)} .
$$

Substituting formula (12) into average evolution dynamic equation (11), the logit dynamic evolution equation can be obtained as follows:

$$
f_{x_{i}}^{\prime}=\frac{e^{E_{i}}}{\sum_{x \in M}\left(E_{x}\right)}-x_{i},
$$

where $E_{i}$ indicates the expected payoff function of travelers choosing travel mode $i$ and $E_{x}$ indicates the expected payoff of travelers choosing travel mode $x$.

Substituting the payoff function into the logit dynamic evolution equation, the logit dynamic model of travelers' travel mode can be obtained:

$$
\frac{\mathrm{d} f_{x}}{\mathrm{~d} t}=\frac{1}{1+e^{\left[\left(\pi_{11}-\pi_{12}\right) x+\pi_{12}-\pi_{2}\right]}}-x .
$$

In order to simplify the calculation, let $a=\pi_{11}-\pi_{12}, b=\pi_{12}-\pi_{2}$. $a$ indicates the payoff difference of travelers choosing air travel when the opponent of the game chooses air and HSR, and in the actual system, it represents the impact of the rapid development of HSR on air. $b$ indicates the payoff difference of travelers choosing air and HSR travel when the opponent of the game chooses HSR, and in the actual system, it represents the travel difference determined by the nature of different modes of transportation, which is affected by travel time, travel comfort, and scientific and technological advancement. 
Therefore, the logit dynamic model of travelers' travel mode selection is

$$
\frac{\mathrm{d} f_{x}}{\mathrm{~d} t}=\frac{1}{1+e^{a x+b}}-x .
$$

\section{Logit Dynamic Model Evolution and Stability Analysis}

Through solving logit dynamic model (15), its stability is analyzed. If it is in a stable state, it must meet the condition that $x^{\prime}=0$. It is obvious that $x=0$ or $x=1$ cannot satisfy the condition of $x^{\prime}=0$. Through a graphic method, the condition satisfying $x^{\prime}=0$ is obtained. Let equation (15) be equal to zero and reorganized as follows:

$$
\frac{1}{x}-1=e^{a x+b} .
$$

Taking the logarithm of both sides, equation (17) can be obtained and solved by a graphical method. Various situations are shown in Figure 1. According to the different values of $a, b$, the solutions of the equations can be divided into three categories: the unique solution that is $x_{1}$; two solutions that are $x_{1}$ and $x_{2}$; and three solutions that are $x_{1}, x_{2}$, and $x_{3}$ :

$$
\left\{\begin{array}{l}
y_{1}=a x+b, \\
y_{2}=\ln \left(\frac{1}{x}-1\right) .
\end{array}\right.
$$

Further derivatives of equations (17) can be obtained as

$$
\left\{\begin{array}{l}
y_{1}^{\prime}=a, \\
y_{2}^{\prime}=-\frac{1}{x(1-x)},
\end{array}\right.
$$

making

$$
a=-\frac{1}{x(1-x)}, \quad x \in(0,1) .
$$

According to the above equation, when $x<0.5$ and $a<-4$, the coordinates of the tangent points of straight line $y_{1}$ and curve $y_{2}$ are $\left(x_{0}, y_{0}\right)=\left(x_{0}, \ln \left(\left(1 / x_{0}\right)-1\right)\right)$. In this case, the constants of the critical condition with two and three intersecting points are $b_{1}=\left(\left(1 / 1-x_{0}\right)+y_{0}\right), b_{2}=$ $\left(\left(1 / x_{0}\right)-y_{0}\right)$ and $2<b_{1}<b_{2}$.

Evolutionary stable strategy (ESS) indicates that, if the majority of the population choose this strategy, no mutation group of any scale adopting other strategies disturbs this state [39]. In order to further verify the stability of the equilibrium point of the system and according to stability analysis theory of the dynamic system, it can be known that if second derivative $J$ at the equilibrium point is less than 0 , then the obtained equilibrium point is the evolutionary stability point, and that is the evolutionarily stable strategy. Therefore, first continuing to find the derivative of formula (15), we can get formula (20), and then combining formula
(16), the derivative of the logit dynamic model can be simplified to

$$
J=\frac{\mathrm{d} f_{x}^{\prime}}{\mathrm{d} x}=f_{x}^{\prime \prime}=-\left[1+\frac{a e^{a x+b}}{\left(1+e^{a x+b}\right)^{2}}\right] .
$$

In addition, using $(1 / x)-1$ instead of $e^{a x+b}$, the derivative of the logit dynamic model can be simplified to

$$
\begin{aligned}
-\left[1+\frac{a((1 / x)-1)}{(1+(1 / x)-1)^{2}}\right] & =-\left[1+\frac{a((1-x) / x)}{(1 / x)^{2}}\right] \\
& =a x(x-1)-1 .
\end{aligned}
$$

From the above formula, as long as $(1 /(x(x-1)))<a$, then $J<0$, and the stability of this point can be further explored. The equilibrium point and stability of the logit dynamic model are shown in Table 2 .

Through the above analysis, the different values of $a, b$ divide the system into the three following categories: there are a unique stable equilibrium point, a stable equilibrium point and an unstable equilibrium point, and two stable equilibrium points and an unstable point. The corresponding system phase diagram is shown in Figure 2.

As $x_{1}^{*}$ can be seen from Figure 1, when $a>0$, equations have one unique solution, $x^{*}$. According to the equilibrium point and stability of the logit dynamic model, $J<0, x^{*}$ is the evolutionary stable strategy point of the system, and only Figure 2(a) in the phase diagram of the logit dynamic system meets the condition. In the logit model, parameters $a, b$ directly cause the change of the evolutionary and stable equilibrium point of the system that, by analyzing parameters $a, b$, can provide the basis for travelers' choice of travel mode and further HSR-air development with HSR speed-up. If HSR speeds up, the equilibrium point of Figure 2(a) moves to the right because the ticket price of HSR with the travel distance of OD is rigid, and travel time is shortened when the ticket price is not volatile, which can reduce the time cost of travelers. Thus, the value of $a, b$ is reduced. In this case, the number of travelers choosing HSR increases. If air travel wants to reduce the loss of passengers, it must reasonably adjust ticket prices and improve its services. Parameter $b$ represents the payoff difference in travel determined by the inherent characteristics of travel mode. With HSR speed-up, air-travel operators can increase the payoff of travel by reducing ticket prices and improving services. At the same time, HSR could promote the road-feeder service before and after the journey, so travelers can enjoy better services and show more public praise. Therefore, from the evolutionary game perspective, if air travel wants to slow down the loss of passenger flow under the condition of HSR speed-up, it must reduce ticket prices and improve services. If HSR wants to keep attracting more passengers, it must also set reasonable ticket prices while remaining a good service.

\section{Discussion}

In order to further explore the game of travelers choosing HSR and air travel with HSR speed-up, a MATLAB 

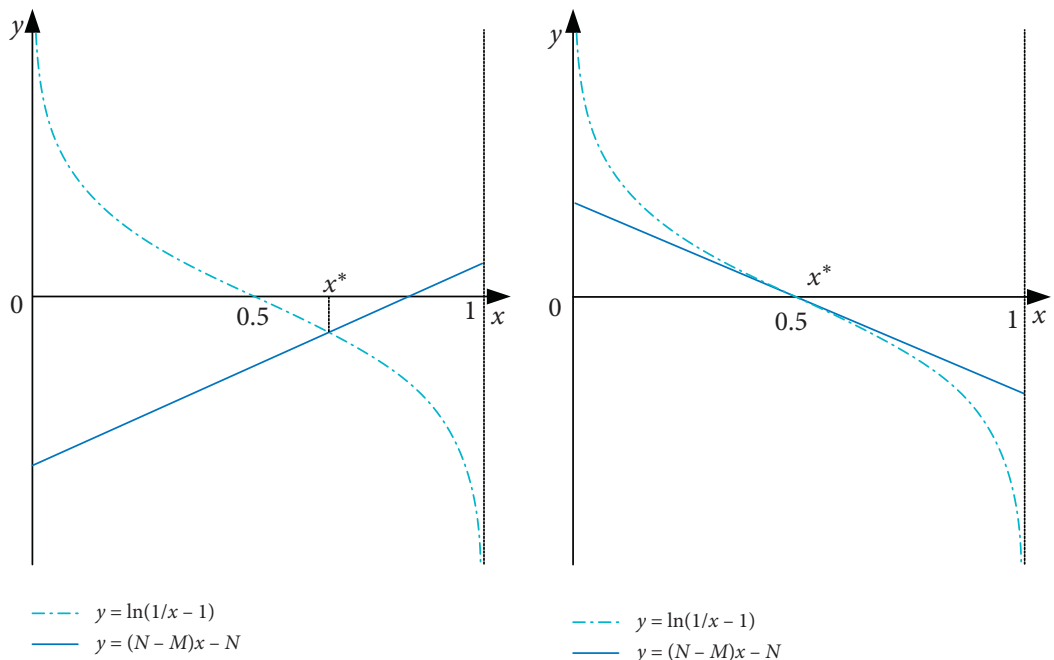

(a)

(b)
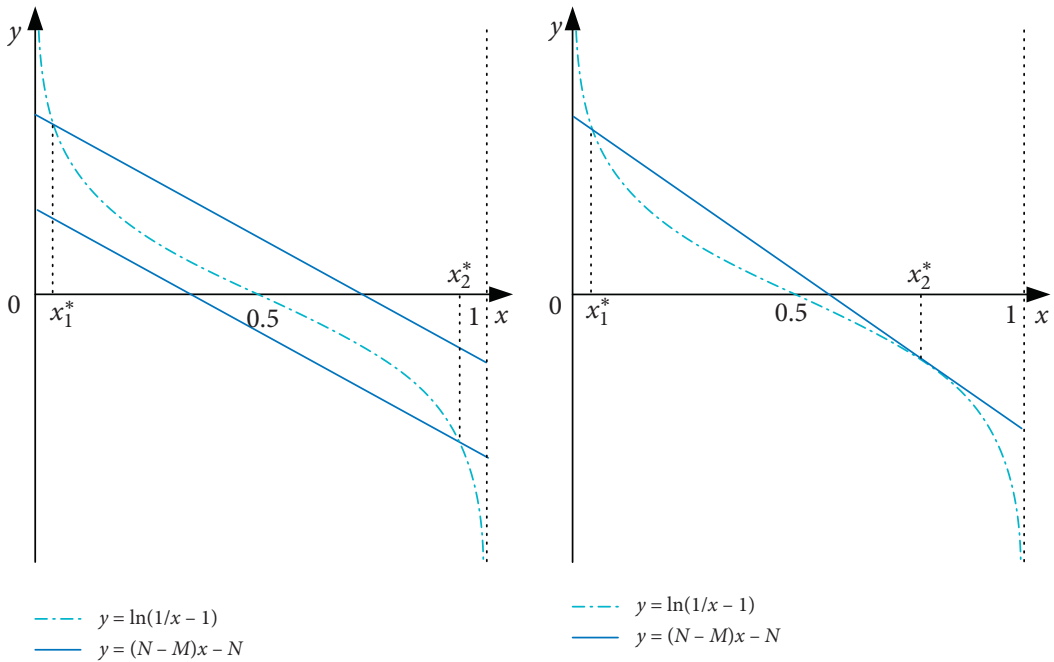

$-\cdot \cdot y=\ln (1 / x-1)$

$y=(N-M) x-N$

(c)

(d)

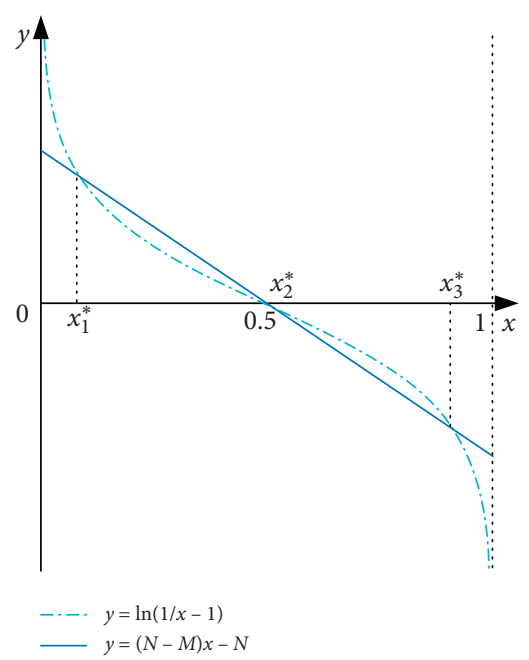

(e)

Figure 1: Logit dynamic choice model. (a) Unique solution: $a>-4$. (b) Unique solution: $a=-4$. (c) Unique solution: $a<-4, b<b_{1}, b>b_{2}$. (d) Two solutions: $a<-4, b=b_{1}, b=b_{2}$. (e) Three solutions: $a<-4, b_{1}<b<b_{2}$. 
TABLe 2: Equilibrium point and stability analysis of the logit dynamic model.

\begin{tabular}{lcccc}
\hline Values of $a, b$ & Number of equilibrium points & Range of equilibrium points & Value of $J$ & Stability \\
\hline$a>-4, b \in R$ & 1 & $x \in(0,1)$ & $J<0$ & Stable \\
\hline$a>-4, b=2$ & 1 & $x=0.5$ & Critical \\
\hline$a>-4, b \neq 2$ & 1 & $x \in(0,0.5) \cup(0.5,1)$ & $J<0$ & Stable \\
\hline$a<-4, b \in\left(-\infty, b_{1}\right) \cup\left(b_{2},+\infty\right)$ & 1 & $x \in\left(0, x_{0}\right) \cup\left(1-x_{0}, 1\right)$ & $J<0$ & Stable \\
\hline$a<-4, b \in\left\{b_{1}, b_{2}\right\}$ & 2 & $x_{1}=x_{0}$ & $J_{1}=0$ & Critical \\
\hline$a<-4, b_{1}<b<b_{2}$ & \multirow{2}{*}{$\begin{array}{c}x_{2} \in\left(0, x_{0}\right) \cup\left(1-x_{0}, 1\right) \\
\text { Stable }\end{array}$} \\
\hline
\end{tabular}

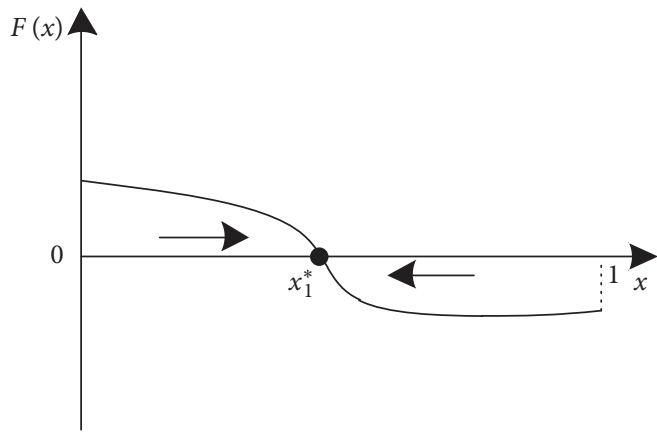

(a)

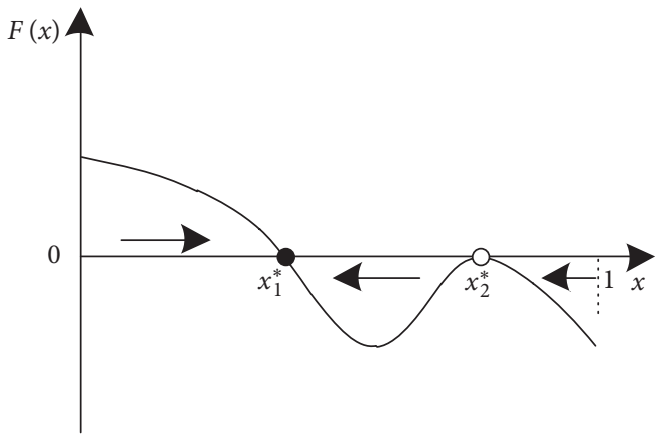

(c)

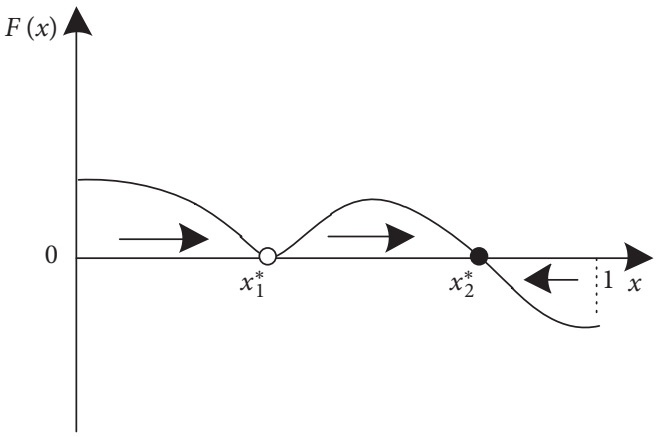

(b)

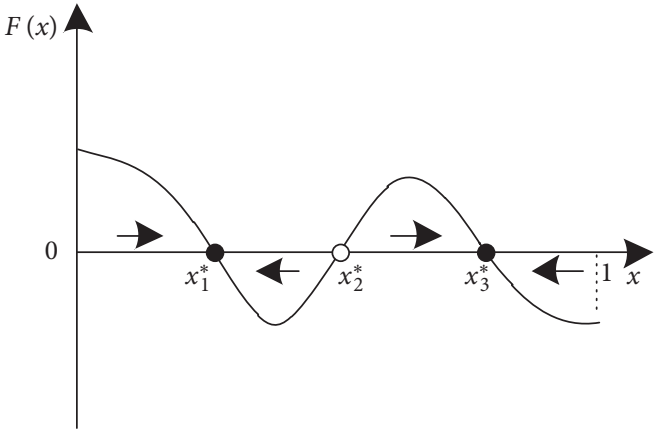

(d)

Figure 2: Phase diagram of the logit dynamic system. (a) One stable point. (b) One stable point and one unstable point. (c) One stable point and one unstable point. (d) Two stable point and one unstable point.

numerical simulation was adopted on the model. The paper assumes that there are only the transportation modes of HSR and air travel between the OD points, and travelers' payoff is the obtained value from travel minus time cost and ticket price. Since travelers are all of the same group, the final payoff of whichever mode of travel they choose is the same; that is,

$$
\pi_{i}=\mu-t_{i}-\frac{m_{i}}{\gamma}
$$

where $\pi_{i}$ represents the final payoff of travelers choosing travel mode $i$; $\mu$ represents the value gained by travelers after the travel, which can be assumed to be the same from the above analysis; $t_{i}$ represents the travel time of travelers choosing the travel mode $i$, which is $S_{i} / V_{i}\left(S_{i}\right.$ represents the distance between OD points, and $V_{i}$ represents the running speed of the selected transportation mode $i) ; m_{i}$ represents the ticket price of travel mode $i$ chosen by travelers, and $\gamma$ indicates the unit time cost. According to the latest research, it can be assumed to $100(i=1,2)[40]$.

4.1. Dynamic Evolution Analysis of Travelers' Travel Mode. Taking the travel time and ticket price of HSR and air travel between the OD points of Beijing and Shanghai as reference benchmarks, the payoff is converted into time variables for further analysis: assuming the value payoff of travelers completing the travel is $\mu \mathrm{h}$; the HSR ticket price is rigid and remains 550 yuan, and travel time is $5.3 \mathrm{~h}$. Air-travel ticket prices are volatile, their average value is 970 yuan, and travel time is $2 \mathrm{~h}$. In order to explore the travel evolution process of 
travelers with HSR speed-up, three scenarios with HSR running speed of 300,400 , and $600 \mathrm{~km} / \mathrm{h}$, respectively, were set for comparative analysis.

Scenario 1. When HSR running speed is $300 \mathrm{~km} / \mathrm{h}$, the final payoff of travelers choosing HSR is $\pi_{2}=\mu-5.3-5.5 \mathrm{~h}$; when both sides of the game choose air to travel, the increase in air-passenger flow leads to a corresponding increase in ticket prices that is equivalent to $0.5 \mathrm{~h}$, and total travel payoff $\pi_{11}=\mu-2-10.2 \mathrm{~h}$; when the opponent of the game chooses HSR and travelers choose air, an air operating company appropriately attracts travelers by lowering ticket prices, equivalent to $0.5 \mathrm{~h}$, and the total payoff of travelers $\pi_{12}=\mu-2-9.2 \mathrm{~h}$. The game payoff matrix of Scenario 1 is shown in Table 3, and the dynamic evolution of travelers' travel mode is shown in Figure 3. The evolution stable value is $(0.6645,0.3355)$.

Scenario 2. When HSR running speed is $400 \mathrm{~km} / \mathrm{h}$ after speed-up, the travel time of travelers choosing HSR is reduced, equivalent to $1.3 \mathrm{~h}$, and final travel payoff is $\pi_{2}=\mu-4-5.5 \mathrm{~h}$; when both sides of the game choose air to travel, the increase of air-travel passenger flow leads to a corresponding increase in ticket price. However, due to HSR speed-up, the increase rate of ticket price is reduced, equivalent to $0.4 \mathrm{~h}$, and total travel payoff is $\pi_{11}=\mu-2-10.1 \mathrm{~h}$; when the opponent of the game chooses HSR and travelers choose air travel, the air operating company lowers ticket prices to attract passengers, equivalent to $1 \mathrm{~h}$ with HSR speed-up, and the total payoff of travelers at this time is $\pi_{12}=\mu-2-8.7$ : The game payoff matrix of Scenario 2 is shown in Table 4, and the dynamic evolution of travelers' travel mode is shown in Figure 4 . The evolution stable value is $(0.4169,0.5831)$.

Scenario 3. When the running speed of the HSR is $600 \mathrm{~km} / \mathrm{h}$ after speed-up, the cost of travel-time reduction of travelers choosing HSR is equivalent to $2.6 \mathrm{~h}$, and the final travel payoff is $\pi_{2}=\mu-2.7-5.5 \mathrm{~h}$; when both sides of the game choose air to travel, the increase of air-passenger flow leads to a corresponding increase in air ticket prices. However, with the influence of HSR speed-up, the increase rate of ticket price is also reduced, which is equivalent to $0.1 \mathrm{~h}$, and total travel payoff is $\pi_{11}=\mu-2-9.8 \mathrm{~h}$; when opponents of the game choose HSR and travelers choose air travel, the air operating company appropriately lowers ticket prices to attract travelers. Under the pressure of HSR speed-up, ticket-price reduction is equivalent to $2 \mathrm{~h}$, and the total payoff of travelers at this time $\pi_{12}=\mu-2-7.7 \mathrm{~h}$. The game payoff matrix of Scenario 3 is shown in Table 5, and the dynamic evolution of travelers' travel mode is shown in Figure 5. The evolution stable value is $(0.2727,0.7273)$.

Through comparative analysis of the three above scenarios, under the condition of HSR speed-up, the proportion of air travel gradually decreases, and HSR becomes the preferred mode for travelers. When HSR running speed is $400 \mathrm{~km} / \mathrm{h}$, the travel-time cost of travelers is reduced, making the proportion of travel by HSR gradually increase from $33.55 \%$ to $58.31 \%$, and the proportion of air travel
Table 3: Game payoff matrix of Scenario 1.

\begin{tabular}{lcc}
\hline Travel mode & Air & High-speed rail (HSR) \\
\hline Air & $(\mu-12.2, \mu-12.2)$ & $(\mu-11.2, \mu-10.8)$ \\
HSR & $(\mu-10.8, \mu-11.2)$ & $(\mu-10.8, \mu-10.8)$ \\
\hline
\end{tabular}

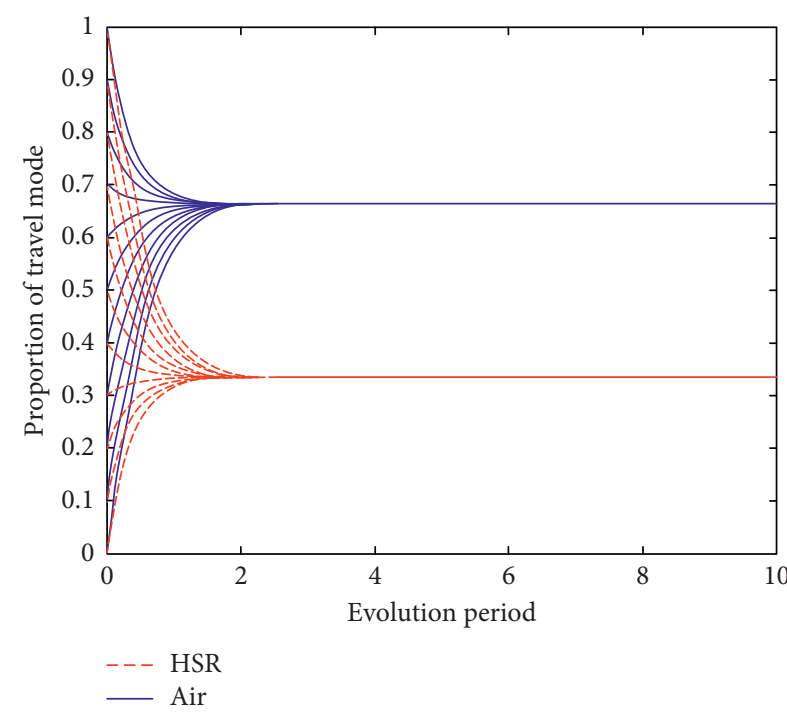

Figure 3: Dynamic evolution of travelers' travel mode under $300 \mathrm{~km} / \mathrm{h}$.

TABle 4: Game payoff matrix of Scenario 2.

\begin{tabular}{lcc}
\hline Travel mode & Air & HSR \\
\hline Air & $(\mu-12.1, \mu-12.1)$ & $(\mu-10.7, \mu-9.5)$ \\
HSR & $(\mu-9.5, \mu-10.7)$ & $(\mu-9.5, \mu-9.5)$ \\
\hline
\end{tabular}

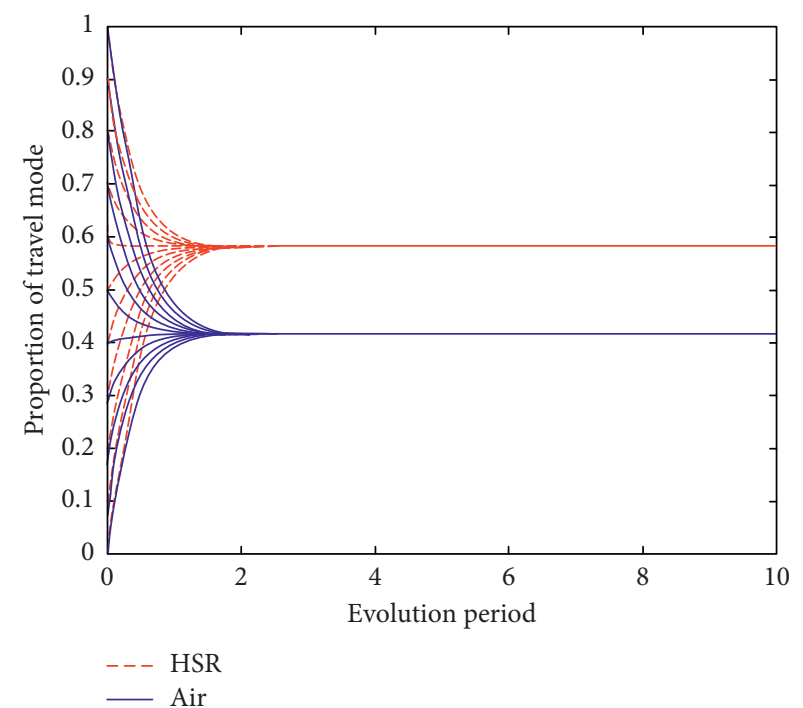

FIGURE 4: Dynamic evolution of travelers' travel mode under 400 $\mathrm{km} / \mathrm{h}$.

gradually decreases from $66.45 \%$ to $41.69 \%$. When HSR running speed is $600 \mathrm{~km} / \mathrm{h}$, the travel-time cost of travelers is reduced, making the proportion of travel by HSR gradually increase from $58.31 \%$ to $72.73 \%$, and the proportion of travel by air gradually decreases from $41.69 \%$ to $27.27 \%$. When 
TABLe 5: Game payoff matrix of Scenario 3.

\begin{tabular}{lcr}
\hline Travel mode & Air & HSR \\
\hline Air & $(\mu-11.8, \mu-11.8)$ & $(\mu-9.7, \mu-8.2)$ \\
HSR & $(\mu-8.2, \mu-9.7)$ & $(\mu-8.2, \mu-8.2)$ \\
\hline
\end{tabular}

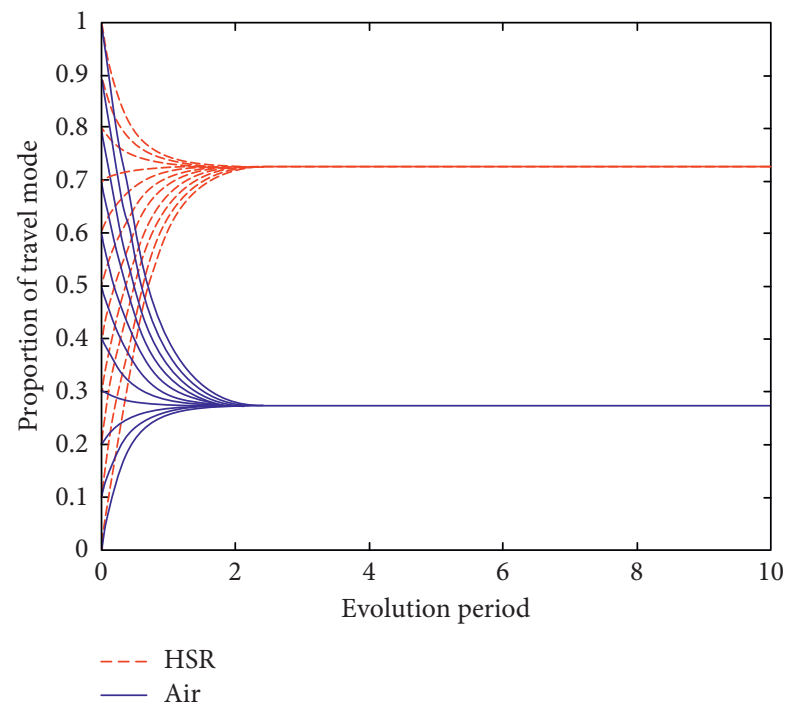

Figure 5: Dynamic evolution of travelers' travel mode under $600 \mathrm{~km} / \mathrm{h}$.

TABLE 6: Relevant parameters of HSR and air travel.

\begin{tabular}{lcccrc}
\hline Parameter & $\alpha_{i}$ & $m_{i}$ & $S_{i} / V_{i}$ & $\gamma$ & $\varepsilon_{i}$ \\
\hline HSR & 0.99 & 553 & - & 100 & 0.7 \\
Air & 0.97 & 970 & $<3$ & 100 & 1 \\
\hline
\end{tabular}

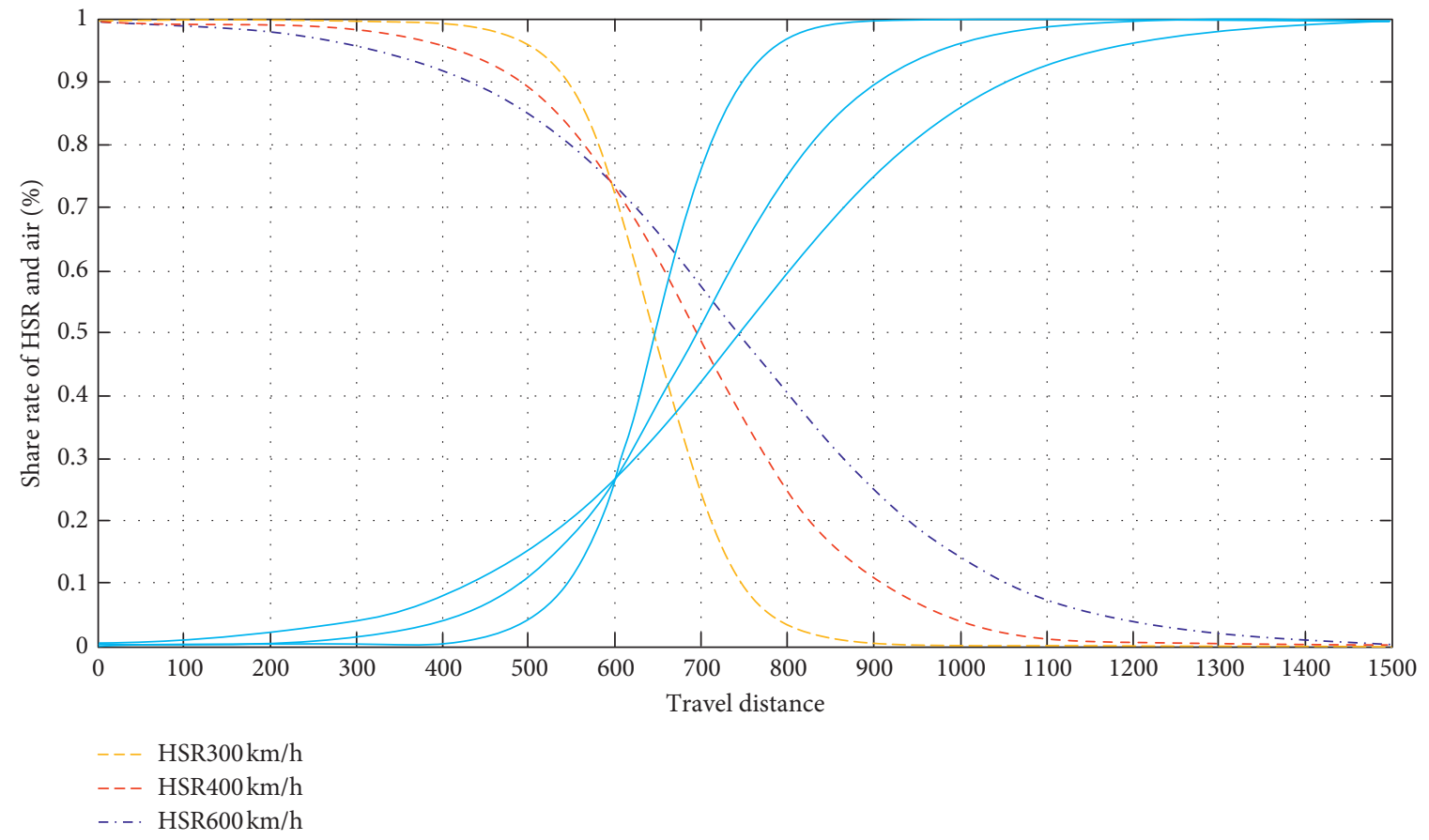

FIgURE 6: Evolution of HSR-air competition. 
HSR running speed increased by $100 \mathrm{~km} / \mathrm{h}$, that is, from 300 to $400 \mathrm{~km} / \mathrm{h}$, the proportion of travelers increased by $24.76 \%$. However, when the speed increased from 400 to $600 \mathrm{~km} / \mathrm{h}$, the proportion of travelers increased by $14.42 \%$. Though speed-increase rates were double, the increase rates of travelers decreased by nearly half. That is because when speed increased from 300 to $400 \mathrm{~km} / \mathrm{h}$ and from 400 to $600 \mathrm{~km} / \mathrm{h}$, there was a decrease of marginal utility of HSR speed-up.

4.2. Evolution Analysis of Competition Range between HSR and Air Travel. The above research proves that, with HSR speed-up, HSR attracts more travelers, and the source of passengers for air travel is further squeezed. The research showed that HSR speed is still slower than that of air travel after HSR speed-up, but it can extend its advantages and compete with air travel in longer travel distances. The evolution of the range of competition with HSR speed-up is further explored by quantitative analysis in the following part of the paper, which studies the change of the share rate of HSR and air travel with the change of travel distance. For a better description, the parameters of safety and environment are introduced in the study, and $\pi_{i}$ is converted into utility function [7]:

$$
\pi_{i}=\alpha_{i}\left(\frac{\mu-S_{i}}{V_{i}-m_{i} / \gamma}\right) \varepsilon_{i} .
$$

The logit share model in the HSR-air game was set as follows [41]:

$$
P(i)=\frac{e^{\pi_{i}}}{\sum_{i=1}^{n} e^{\pi_{i}}},
$$

where $P(i)$ indicates the probability of choosing travel mode $i$; $n$ indicates the provided travel mode; and $\pi_{i}$ is a utility function. The parameters in formulas (23) and (24) take the data of Beijing-Shanghai HSR and air travel as analysis objects, and previous studies were reviewed [42, 43]. The quantitative research indices are shown in Table 6, and the evolution curve of the share model is shown in Figure 6.

Through MATLAB numerical simulation, the change of the market-share rate of air and HSR travel under the condition of HSR speed-up was obtained, as shown in Figure 6. When HSR speed increased from 300 to $600 \mathrm{~km} / \mathrm{h}$, the intersection of the two curves obviously moved to the right, indicating that the HSR comparative advantage range was extended, the HSR market share increased, the absolute advantage range of air travel shifted to longer-distance travel, and HSR-air competition range expanded.

\section{Conclusion}

On the basis of analysis of HSR speed-up, the paper systematically summarized the operation and development of HSR and air, established a logit dynamic evolution game model on the basis of the bounded rationality of travelers, and obtained the equilibrium point and stability of travelers' choice in the process of system evolution by using mathematical analysis methods. Finally, through data simulation, changes in the share rate of HSR and air travel, and further expansion of the competition range is obtained. Conclusions are as follows:

(1) There is a unique stable strategy for the evolution of travel modes. Under the bounded rationality of travelers, the impact of HSR speed-up on travelers' travel modes is slowly progressive. Travelers are eventually more likely to choose HSR after continuously improving their strategies. In this process, it is better for the two operating companies to adjust their services in time and make relative preparations.

(2) With the increase of speed in the same range, the evolution process has different convergence, which indicates that, besides a single variable (speed, ticket price, etc.), the service of HSR and air travel has great influence on traveler choices. The competition range expands to longer-distance travels, the range in which HSR has a comparative advantage extends, and air travel is influenced as its market share shrinks.

(3) The ticket price of air travel is relatively volatile. As HSR gradually dominates the market, it is of great importance for airlines to provide better services and cheaper tickets to keep their customers.

In MATLAB simulation, the selected data were not adequate enough. Real-time big data to further analyze the development of HSR and air travel by the logit dynamic evaluation of the evolutionary game and the evolution of travelers with the change of ticket prices are topics that require further research.

\section{Data Availability}

The simulation data used to support the findings of this study are available upon request.

\section{Conflicts of Interest}

The authors declare no conflicts of interest.

\section{Acknowledgments}

This study was supported by the Humanities and Social Science Funds of the Ministry of Education (grant no. 19YJC630124) and Quality Promotion Plan Program of Postgraduate Education of Shandong Province (grant no. SDYKC18081).

\section{References}

[1] https://wenku.baidu.com/view/13dbe9993d1ec5da50e2524de 518964bce84d2f0.html.

[2] C. Behrens and E. Pels, "Intermodal competition in the London-Paris passenger market: high-speed rail and air transport," Journal of Urban Economics, vol. 71, no. 3, pp. 278-288, 2012. 
[3] Q. Zhang, H. Yang, Q. Wang, and A. Zhang, "Market power and its determinants in the Chinese airline industry," Transportation Research Part A: Policy and Practice, vol. 64, pp. 1-13, 2014.

[4] Y. Wan, H.-K. Ha, Y. Yoshida, and A. Zhang, "Airlines' reaction to high-speed rail entries: empirical study of the Northeast Asian market," Transportation Research Part A: Policy and Practice, vol. 94, pp. 532-557, 2016.

[5] K. E. Haynes, "Labor markets and regional transportation improvements: the case of high-speed trains," The Annals of Regional Science, vol. 31, no. 1, pp. 57-76, 1997.

[6] Y. Park and H.-K. Ha, "Analysis of the impact of high-speed railroad service on air transport demand," Transportation Research Part E: Logistics and Transportation Review, vol. 42, no. 2, pp. 95-104, 2006.

[7] J. Luo, H. Kuang, T. Feng, and D. Song, "Research on the coopetition between high speed rail and air based on two stage game model," Systems Engineering-Theory \& Practice, vol. 39, no. 1, pp. 150-164, 2019.

[8] W. Wang, H. Sun, and J. Wu, "How does the decision of highspeed rail operator affect social welfare? considering competition between high-speed rail and air transport," Transport Policy, vol. 88, pp. 1-15, 2020.

[9] Z. Chen, "Impacts of high-speed rail on domestic air transportation in China," Journal of Transport Geography, vol. 62, pp. 184-196, 2017.

[10] X. Ke, H. Chen, Y. Hong, and C. Hsiao, "Do China's highspeed-rail projects promote local economy?-new evidence from a panel data approach," China Economic Review, vol. 44, pp. 203-226, 2017.

[11] Y. Lin, "Travel costs and urban specialization patterns: evidence from China's high speed railway system," Journal of Urban Economics, vol. 98, pp. 98-123, 2017.

[12] S. Shao, Z. Tian, and L. Yang, "High speed rail and urban service industry agglomeration: evidence from China's Yangtze River delta region," Journal of Transport Geography, vol. 64, pp. 174-183, 2017.

[13] H. Yang, G. Burghouwt, J. Wang, T. Boonekamp, and M. Dijst, "The implications of high-speed railways on air passenger flows in China," Applied Geography, vol. 97, pp. 1-9, 2018.

[14] M. Ivaldi and C. Vibes, "Price competition in the intercity passenger transport market: a simulation model," Journal of Transport Economics and Policy, vol. 42, no. 2, pp. 225-254, 2008.

[15] N. Adler, E. Pels, and C. Nash, "High-speed rail and air transport competition: game engineering as tool for costbenefit analysis," Transportation Research Part B: Methodological, vol. 44, no. 7, pp. 812-833, 2010.

[16] A. Zhang, Y. Wan, and H. Yang, "Impacts of high-speed rail on airlines, airports and regional economies: a survey of recent research,” Transport Policy, vol. 81, pp. A1-A19, 2019.

[17] R. Zhang, D. Johnson, W. Zhao, and C. Nash, "Competition of airline and high-speed rail in terms of price and frequency: empirical study from China," Transport Policy, vol. 78, pp. 8-18, 2019.

[18] C.-H. Wen, W.-C. Wang, and C. Fu, "Latent class nested logit model for analyzing high-speed rail access mode choice," Transportation Research Part E: Logistics and Transportation Review, vol. 48, no. 2, pp. 545-554, 2012.

[19] D. McFadden, Conditional Logit Analysis of Qualitative Choice Behavior, Academic Press, New York, NY, USA, 1974.

[20] K. E. Train, Discrete Choice Methods with Simulation, Cambridge University Press, Cambridge, UK, 2009.
[21] S.-Y. Jung and K.-E. Yoo, "Passenger airline choice behavior for domestic short-haul travel in South Korea," Journal of Air Transport Management, vol. 38, pp. 43-47, 2014.

[22] S. Krygsman, T. Arentze, and H. Timmermans, "Capturing tour mode and activity choice interdependencies: a co-evolutionary logit modelling approach," Transportation Research Part A: Policy and Practice, vol. 41, no. 10, pp. 913-933, 2016.

[23] P. C. Fishburn, Utility Theory for Decision-Making, Wiley Press, New York, NY, USA, 1970.

[24] M. Ernst, Population, Species, and Evolution, Belknap Press of Harvard University Press, Boston, MA, USA, 1970.

[25] X. Lin, Research of Travel Choice Behavior for Inter-City Trips under Highway Toll Regulation on Holiday - In the Case of the Spring Festival Holiday, Beijing Jiaotong University, Beijing, China, 2019.

[26] H. Yang and A. Zhang, "Effects of high-speed rail and air transport competition on prices, profits and welfare," Transportation Research Part B: Methodological, vol. 46, no. 10, pp. 1322-1333, 2012.

[27] T. D’Alfonso, C. Jiang, and V. Bracaglia, "Would competition between air transport and high-speed rail benefit environment and SW," Transportation Research Part B, vol. 74, pp. 118-137, 2015.

[28] Q. Bian, L. Song, B. Mao, and X. Jiang, "High speed rail and air transport competition: a market-entry game approach," Journal of Transportation Systems Engineering and Information Technology, vol. 19, no. 5, pp. 20-27, 2019.

[29] T. D’Alfonso, C. Jiang, and V. Bracaglia, “Air transport and high-speed rail competition: environmental implications and mitigation strategies," Transportation Research Part A, vol. 92, pp. 261-276, 2016.

[30] K. Wang, W. Xia, A. Zhang, and Q. Zhang, "Effects of train speed on airline demand and price: theory and empirical evidence from a natural experiment," Transportation Research Part B: Methodological, vol. 114, pp. 99-130, 2018.

[31] A. Mukhopadhyay and S. Chakraborty, "Periodic orbit can be evolutionarily stable: case study of discrete replicator dynamics," Journal of Theoretical Biology, vol. 497, Article ID 110288, 2020.

[32] W. Xia and A. Zhang, "High-speed rail and air transport competition and cooperation: a vertical differentiation approach," Transportation Research Part B: Methodological, vol. 94, pp. 456-481, 2016.

[33] Q. Zhang, H. Yang, and Q. Wang, "Impact of high-speed rail on China's big three airlines," Transportation Research Part A: Policy and Practice, vol. 98, pp. 77-85, 2017.

[34] H. Xiao, Game Theory Approach to Some Problems in Transportation Planning, Wuhan University, Wuhan, China, 2010.

[35] W. H. Sandholm, Population Games and Evolutionary Dynamics, MIT Press, London, UK, 2010.

[36] Z. Wang, Q. Wang, B. Chen, and Y. Wang, "Evolutionary game analysis on behavioral strategies of multiple stakeholders in e-waste recycling industry," Resources, Conservation and Recycling, vol. 155, Article ID 104618, 2020.

[37] D. Fudenberg and J. Tirole, Game Theory, MIT Press, Cambridge, MA, USA, 1991.

[38] J. F. Nash, "Equilibrium points in $\mathrm{N}$-person games," Proceedings of the National Academy of Sciences, vol. 36, no. 1, pp. 48-49, 1950.

[39] M. Oskar and V. N. John, Theory of Games and Economic Behavior, Princeton University Press, Princeton, NJ, USA, 1980.

[40] http://www.stats.gov.cn/tjsj/ndsj/2019/indexch.htm. 
[41] B. Ma, Y. Zhang, and C. Zhao, "Estimation of distributing rates of high-speed passenger flows with the logit model," Journal of Beijing Jiaotong University, vol. 2, pp. 66-69, 2003.

[42] J. Ding, F. Jin, and J. Wang, "Competition game of high-speed rail and civil aviation and its spatial effect-a case study of Beijing-Shanghai high-speed rail," Economic Geography, vol. 33, no. 5, pp. 104-110, 2013.

[43] D. Li, Research on Mode Split between HSR and Civil Aviation of Transport Corridor, Southeast University, Nanjing, China, 2015. 